Sociologie et sociétés

\title{
Partir du droit pour aborder le problème de l'économie
} informelle

Réflexions sur le projet d'une norme internationale à travers deux " économies informelles " au Togo

A legal perspective on the issue of the informal economy Thoughts regarding the project of an international standard based on the case of two "informal economies" in Togo

\section{Élise Panier}

Volume 47, numéro 1, printemps 2015

Travail et informalité : nouvelles figures de l'exploitation et des mobilisations au nord et au sud

Work and the informal sector: new figures of exploitation and mobilisations in the north and south

\section{URI : https://id.erudit.org/iderudit/1034420ar}

DOI : https://doi.org/10.7202/1034420ar

Aller au sommaire du numéro

\section{Éditeur(s)}

Les Presses de l’Université de Montréal

\section{ISSN}

0038-030X (imprimé)

1492-1375 (numérique)

Découvrir la revue

\section{Citer cet article}

Panier, É. (2015). Partir du droit pour aborder le problème de l'économie informelle : réflexions sur le projet d'une norme internationale à travers deux " économies informelles » au Togo. Sociologie et sociétés, 47(1), 97-120. https://doi.org/10.7202/1034420ar
Résumé de l'article

En partant du récent projet de norme de l'OIT visant à favoriser la formalisation de l'économie informelle, cet article propose d'interroger la démarche institutionnelle à travers une réflexion sur le droit comme critère d'intelligibilité de la catégorie de l'informel. Sur la base d'une enquête réalisée au Togo, cette contribution présente les modalités nombreuses et complexes des manifestations du droit du travail et du droit de l'activité économique dans les deux espaces d'informalité du travail que sont la zone franche d'exportation et le secteur dénommé informel au Togo. Entre surformalisation spécifique et im-pénétration publique ou syndicale dans un cas, et intervention publique et syndicale relativement quotidienne dans l'autre, la manière de vouloir faire de l'économie informelle un objet d'intervention global, en distinguant l'emploi informel des unités économiques, n'est pas sans soulever des questions sur la pertinence de la démarche et le rôle de ce concept souvent critiqué mais, paradoxalement, d'usage permanent. 


\title{
$\geqslant$
}

\section{Partir du droit pour aborder le problème de l'économie informelle}

Réflexions sur le projet d'une norme internationale à travers deux «économies informelles » au Togo

\section{ÉLISE PANIER}

CRIMT

Université de Montréal

C.P. 6128 , Succursale Centre-ville

Montréal QC Canada $\mathrm{H}_{3} \mathrm{C}_{3} / 7$

Courriel : elise.panier@umontreal.ca

\section{INTRODUCTION}

\begin{abstract}
A
U DÉBUt DES ANNÉES 1970, le «secteur informel» désignait une économie urbaine dynamique, à petit capital et petite échelle, la source possible d'une richesse à venir dans les pays en développement (BIT, 1972: 4 et Hart, 1973). Des politiques de formalisation du secteur informel vont toutefois être promues dans les années 1980 en s'appuyant sur le constat d'un secteur économique improductif (Lautier, 2004) ou, pour certains, sur une vision «misérabiliste» du phénomène (Bangasser, $2000: 14$ ). Mais l'informel va commencer à poser un dilemme à l'Organisation internationale du travail (OIT) au début des années 1990. Il s'agissait alors de savoir, selon les termes de ce dilemme, s'il fallait promouvoir ce secteur ayant sa propre réalité ou le faire plutôt entrer dans le champ des règles qui déterminent la formalité de l'activité, et ce, au risque des pertes d'emploi (BIT, 1991). Or, aujourd'hui, le titre du récent projet de recommandation de l'OIT semble indiquer que l'économie informelle ne peut plus désormais constituer une alternative (Lautier, 2003). Après plus de quarante ans d'évolution de l'approche sous-jacente à la notion d'informel, l'OIT est ainsi sur le point d'adopter un instrument normatif visant à aider les gouvernements, du Nord comme du
\end{abstract}


Sud, à «faciliter la transition de l'économie informelle vers l'économie formelle» (CIT, 2014: 1) ${ }^{1}$.

Le choix de ce thème en vue de l'adoption d'une norme dans le cadre de la Conférence internationale du travail (ci-après CIT) est issu d'une proposition du groupe des employeurs à l'OIT appuyée par celui des travailleurs ${ }^{2}$. Au début du travail de la commission tripartite ${ }^{3}$ réunie à cette fin pendant la CIT en 2014, chaque groupe a présenté une vision des enjeux du projet et des moyens à mobiliser. Le représentant des employeurs a ainsi déclaré que «le moment est venu de transformer l'économie informelle et de la mettre davantage au service de la communauté mondiale», notamment en «débloqu [ant] la masse de capital détenue par les populations pauvres du monde» (CIT, 2014: 4). Pour ce dernier, «l'approche fondée sur les droits» ne doit pas consister en « une simple extension des droits» mais être assortie d'une formalisation adaptée et progressive des unités économiques. Le représentant du groupe des travailleurs insistant de son côté sur le sous-développement comme cause première de l'économie informelle et sur la nécessité «d'une mutation structurelle vers une production à plus forte valeur ajoutée», préconise plutôt une «action immédiate» en matière de droits sociaux.

Il ressort de ces deux interventions que les droits et la formalisation peuvent être aussi bien synonymes qu'antagoniques. Et c'est précisément à partir de cette position instable du droit que nous voudrions interroger ici le projet de recommandation de l'OIT en illustrant notre propos par des recherches effectuées dans le contexte d'un pays d'Afrique de l'Ouest: le Togo.

1. Le projet de préambule contient 8 paragraphes qui énumèrent des aspects négatifs du phénomène. Son ampleur en fait une «entrave de taille aux droits des travailleurs", la plupart des travailleurs n'ont pas le choix d'y entrer, c'est là où les déficits de travail décent sont les plus prononcés, etc. Seul le dernier point mentionne le potentiel de certains travailleurs ou unités qui pourrait être développé. Le préambule de la recommandation 204 «concernant la transition de l'économie informelle vers l'économie formelle», issue du processus et adoptée en juin 2015 à Genève lors de la $104^{\mathrm{e}}$ session de la CIT, est sensiblement similaire.

2. Voir les documents institutionnels du Conseil d'administration du BIT (GB/316/INS/4 par. 23-37, GB/317/PV par. 3-24). Certains représentants préconisaient de simples discussions mais il fut décidé d'aller vers la forme d'une recommandation, type de norme non contraignante prévue si l'objet ne se prête pas à l'adoption immédiate d'une convention (article 19 1. de la constitution de l'OIT).

3. L'OIT fonctionne sur la base d'un dialogue international tripartite entre les gouvernements, les travailleurs et les employeurs. La commission réunie dans le cadre du projet de recommandation a rassemblé aux alentours de 200 participants provenant des différents groupes. Le bureau de la commission a été constitué sur la base d'un membre gouvernemental (Afrique du Sud) à titre de président et de deux viceprésidents, dont un membre employeur (Ghana) et un membre travailleur (Bulgarie). Une deuxième session en commission s'est tenue au mois de juin 2015 sur le même format et a porté sur le projet de texte issu des discussions de 2014 tel que modifié après consultation des membres gouvernementaux (article 36 du règlement de la CIT) et discussions «informelles» (BIT, 2015ab et CIT, 2015). Le projet analysé ici est celui issu de la première session de discussions. Des éléments de la deuxième phase du processus seront mentionnés en note si besoin pour notre propos. 
Dans le champ spécifique des sciences juridiques, l'économie informelle n'est pas

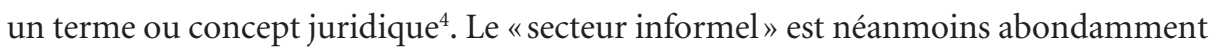
abordé dans ce domaine d'étude et ce, particulièrement en ce qui concerne l'Afrique. Il désigne principalement une donnée qui échappe aux règles officielles ou qui sort du champ d'application du droit mais aussi un espace de pluralisme juridique qui pourrait être pris en charge par certaines dispositions de droit nouvelles ou interprétées ${ }^{5}$. Le droit occupe également une place très importante dans les autres domaines d'utilisation ou de réflexion sur l'informalité, qu'il s'agisse de l'outil descriptif des économies urbaines des pays en développement, de l'objet d'intervention politique locale et internationale, ou de l'objet d'analyse théorique largement usité, critiqué et affiné qu'il est devenu en sciences sociales. Le droit apparaît même central dans la constitution de cette donnée économique et sociale contemporaine dans la mesure où les contours de sa manifestation ne peuvent être dessinés qu'en lien avec l'État (V. par ex. Fontaine et Weber, 2011; Nélisse et al., 1994; Lautier et al., 1991). Dans le projet normatif de l'OIT, le droit est à cet égard partout, constitutif à la fois d'un critère de définition de l'informel et d'un outil important de la formalisation qu'il s'agit de promouvoir.

L'interrogation de cet article portera ainsi principalement sur le lien entre droit et informel au sens où ce n'est pas tant l'économie informelle qu'il s'agira d'interroger sociologiquement que la production ou cristallisation de cette catégorie et la formulation de ses enjeux aujourd'hui à partir du droit dans «l'ensemble de la société $»^{6}$. À partir de recherches effectuées sur le droit au Togo ${ }^{7}$, il s'agira d'illustrer

4. Une recherche rapide dans deux bases de données de revues de droit aboutit à deux types de résultats principaux: l'économie informelle est abordée dans une perspective internationale (pour aborder certains pays en développement ou interroger les normes de responsabilité sociale des entreprises) et l'informel comme qualificatif désigne certaines modalités de production et de mise en œuvre de normes (un droit informel, un contrôle informel, un régime informel, une négociation informelle, etc.).

5. Un récent ouvrage sur le droit africain rassemblant les contributions de juristes francophones d'Afrique et d'ailleurs contient de nombreuses occurrences sur le sujet: il y a «la production informelle du droit écrit», le pluralisme normatif africain, et le «secteur informel» qui «se caractérise par sa non-soumission aux lois de l'État» et «ne recourt au droit de l'État que pour mieux l'assujettir à sa logique» (Cissé, 2014). Dans une étude sur le projet de droit du travail africain de l'OHADA, la question se pose de l'applicabilité du droit du travail au «secteur informel» à partir de son champ d'application. Il n'est pas impossible de qualifier le lien de subordination mais «il peut sembler difficile de rechercher l'indice fondé sur le rapport de pouvoir» du fait de la prépondérance des liens de conjugalité ou de parenté. L'auteur propose alors une réflexion sur le lien de subordination économique pour étendre le champ d'application du droit du travail (Miendjiem, 2014).

6. Bruno Lautier propose de déplacer ainsi le regard dès les années 1990 sur les activités informelles (Lautier, (2013 [1992]). La piste de recherche ouverte par l'auteur, et qui nous intéresse particulièrement ici, est celle du droit à travers la question des rapports à l'État, et plus précisément du type de « compromis juridico-politique» qui fonde l'équilibre entre une production officielle d'un droit inappliqué et des formes normatives mises en œuvre en pratique.

7. Ces recherches s'appuient sur des données documentaires (textes juridiques, décisions judiciaires, documents institutionnels, syndicaux et associatifs, mémoires de l'École nationale d'administration) et des entrevues réalisées avec différents acteurs publics et privés, en particulier de l'administration du travail (18), de la SAZOF (3), du monde judiciaire (9) et syndical (26 dont 3 du côté patronal), du milieu associatif (2) ainsi que des travailleurs et des employeurs (21). Voir Panier, 2014. 
la conceptualisation du projet de norme de l'OIT et d'en questionner l'objectif affiché de favoriser la prise en charge du problème de l'informel. Cette démarche se réalisant dans une interrogation sur le projet international à travers sa dimension «globale» où «la question des sémantiques, le choix des mots et des concepts prennent un relief tout à fait particulier. Comment trouver un accord sur des catégories communes qui permet d'agir et de penser de concert?» (Abélès, 2008: 146).

La formulation de l'approche générale de l'OIT met en évidence un critère de droit tout à fait ambivalent au sein de la dualité formel-informel, qu'il s'agisse d'en énoncer le problème de départ ou de préciser les aspects de sa finalité. Si la complexité qu'embrasse le projet au sujet du droit est confirmée par l'étude des modalités par lesquelles le droit participe à l'informalité du travail au Togo, la comparaison entre le secteur officiellement appelé «informel» et la zone franche d'exportation (ci-après ZFE) soulève aussi, sur le plan politique, la question de l'enjeu du choix du problème général de l'économie informelle comme objet de l'action normative internationale. Après étude dans un premier temps du projet de recommandation internationale au sein duquel le droit occupe une place tout à fait ambiguë, qu'il s'agisse de définir la notion même d'économie informelle ou de déterminer l'objectif de la norme et les outils pertinents pour sa réussite, nous essaierons d'illustrer, dans un second temps, cette ambivalence du droit et l'indétermination de la notion d'informel en comparant deux «économies informelles» repérables au Togo en vertu de la conceptualisation de l'OIT.

\section{L'AMBIVALENCE DU DROIT DANS LE PROJET DE RECOMMANDATION}

INTERNATIONALE POUR FAVORISER LA TRANSITION VERS L'ÉCONOMIE FORMELLE

L'utilisation du droit comme critère de définition générale de l'économie informelle n'est pas nouvelle dans le travail de l'OIT mais la formulation de ce critère général au sein du récent projet rend sa position beaucoup plus instable (I.1.), ce qui favorise alors l'imprécision en droit de sa finalité qu'est l'économie formelle; cette finalité paraissant constituer en même temps une condition préalable à la réussite de ses objectifs (I.2.).

\section{1 Le droit des deux côtés de ce qui délimite l'économie informelle}

La mondialisation ayant conduit à l'amplification du champ de l'informel et, plus particulièrement, à l'informalisation des relations d'emploi avec la multiplication des emplois non conventionnels et atypiques (sous-traitance, travailleurs précaires, externalisation, etc.), les travaux de 2014 de l'OIT cherchent à embrasser l'ampleur de ce qui constitue le phénomène de l'informel qui se manifeste au Nord et au Sud et dans des cadres plus ou moins institutionnalisés selon les divers contextes nationaux. La première section du projet qui définit son champ d'application préconise en effet une application de l'instrument à tous les travailleurs et à toutes les unités économiques de l'économie informelle, seules les activités illicites devant en être exclues. Pour englober cette approche qui distingue l'économie informelle des unités de celle des travailleurs 
(que l'on appelle «l'emploi informel»), une «nouvelle»8 définition générale est proposée: «l'expression «économie informelle» désigne toutes les activités économiques de travailleurs et d'unités économiques qui — dans la législation ou la pratique — ne sont pas couverts ou sont insuffisamment couverts par des dispositions formelles» (CIT, 2014: 148) ${ }^{9}$. Le droit est non seulement central ici mais il est surtout ambivalent, ce qui illustre dans un sens cette volonté d'appréhender l'informel dans la totalité de ce qu'il représente.

En effet, la place possible du droit dans l'informel est rendue plus diversifiée avec cette définition qui ajoute un élément au sein de l'idée d'une informalité «en droit». Si l'expression «en vertu de la législation ou de la pratique» suggérait déjà en 2002 que le droit ne se confondait pas avec ce qui permettait de passer d'un côté ou de l'autre de ces catégories de l'économie, on considérait de manière générale que l'économie informelle se trouvait à l'extérieur des cadres juridiques de protection. Car selon les conclusions adoptées lors de la $90^{\mathrm{e}} \mathrm{CIT}$ sur la base du rapport Travail décent et économie informelle, «l'expression «économie informelle» fait référence à toutes les activités économiques de travailleurs et d'unités économiques qui ne sont pas couverts - en vertu de la législation ou de la pratique — par des dispositions formelles», soit qu'elles n'entrent pas dans le champ d'application de la loi, soit que la loi n'est pas appliquée, soit que la loi n'est pas respectée car inadaptée (CIT, 2002). Le droit renvoyait donc en 2002 à ce qui pouvait permettre de délimiter, en apparence assez facilement ${ }^{10}$, ce qui se trouvait dans le formel ou l'informel, puisqu'il était dans l'ensemble absent. Or, en 2014, la définition proposée ne renvoie plus seulement à un droit inapproprié ou silencieux, donc absent, mais aussi à un droit qui n'est que partiellement là. L'ajout de l'insuffisance de couverture par des dispositions formelles dans les conclusions proposées en $2014^{11}$ donne donc à voir l'utilisation d'un critère général de droit très

8. C'est dans seulement une des trois langues de travail de l'OIT que l'on repère cette nouveauté, d'où l'utilisation des guillemets ici puisque nous nous concentrerons sur l'évolution des approches dans le cadre linguistique du français (voir note 11 infra).

9. Définition figurant désormais au point 2. a) de la recommandation 204 de l'OIT.

10. Le rapport exprime néanmoins à l'époque des incertitudes sur la délimitation de l'économie informelle. Il y a d'un côté les zones grises entre formel et informel et, en plus, «le terme "informel” ne signifie pas qu'il n'existe aucune règle ni norme (...). Nous ignorons, par contre, sur quoi se fondent ces règles ou normes informelles, et si elles respectent les droits fondamentaux des travailleurs et de quelle manière» (BIT, 2002: 4-5).

11. Cet ajout est assez difficile à retracer dans les travaux en français de l'OIT car le caractère insuffisant apparaît en référence à des travaux dont les résolutions ne le contiennent pas. C'est le cas d'un rapport de 2010 (OIT, 2010: 9 et 59) en référence aux conclusions de 2002. Dans le rapport d'experts de 2013 (BIT, 2013: 3), il est indiqué que le porte-parole des travailleurs propose cette approche de définition en s'appuyant sur les travaux de 2003 de la Conférence internationale des statisticiens du travail (CIST). Le critère d'insuffisance est ensuite repris en 2014 dans les questions à soumettre aux membres de l'OIT, en s'appuyant cette fois sur les travaux de 2002 (BIT, 2014a: 63; BIT, 2014b: 20). Or, il ressort de la comparaison des différentes versions linguistiques des travaux du début des années 2000 que si les versions en anglais et en espagnol des résolutions adoptées en 2002 comportent bien cette spécification, la version en français ne sera ajustée, et ce, discrètement, qu'une dizaine d'années plus tard. Toujours est-il que toutes les versions 
ambigu dans la détermination de l'«économie informelle» même s'il appuie la volonté affirmée d'absorber dans cette notion toute la complexité à laquelle elle renvoie.

On remarque également, en ce qui concerne l'enjeu de la démarche de l'OIT, la position instable du droit malgré l'objectif relativement clair, si l'on peut dire, du projet qui est de faire passer l'informel dans le formel en facilitant la transition. Cet objectif général est formulé de la manière suivante au point 12 du texte ${ }^{12}$ :

a) faciliter la transition des travailleurs et des unités économiques de l'économie informelle vers l'économie formelle tout en respectant les droits fondamentaux des travailleurs et en assurant les possibilités de sécurité du revenu, de subsistance et d'entrepreneuriat. (CIT, 2014: 149)

Là encore, la place du droit dans le programme est ambivalente puisqu'il faut veiller à ce que la formalisation ne se fasse pas sans lui. Car il y a le risque d'une formalisation de l'économie informelle qui ne s'appuie pas sur le respect des droits fondamentaux ou qui s'amorce sans reconnaître au préalable l'entrepreneuriat et même la subsistance ${ }^{13}$. La finalité du projet en résulte alors aussi ambiguë car la formalisation, si elle est positive dans son principe, peut également s'avérer partielle, insuffisante, voire étrangère à l'économie formelle recherchée.

\section{2 L'économie formelle à la fois finalité et condition préalable}

Si la formulation de l'objectif de la formalisation est précise, il n'y a pas de définition de l'économie formelle qui sera visée en fin de compte car les moyens à envisager pour prendre en charge l'économie informelle sont très divers, impliquant des stratégies politiques complexes et intégrées, qui combinent le court terme et le long terme, des actions publiques préventives et coercitives, des mesures incitatives et correctives ${ }^{14}$. Lorsque l'on regarde l'ensemble des types de mesures préconisées et leurs objets, il semble que la mise en place d'un cadre pertinent de l'économie formelle doit presque précéder, ou en tout cas accompagner, la transition de l'informel vers le formel.

On remarque dans ce sens que les interventions visant à faciliter la transition de l'économie informelle vers l'économie formelle sont explicitement précisées comme

de l'époque évoquent bien une idée générale d'un droit dans l'ensemble «absent» dont les précisions ne sont pas reprises dans la recommandation.

12. Point 12 qui correspond mot pour mot au point 1. a) de la recommandation 204.

13. Lors des discussions de 2015, la membre gouvernementale de l'Argentine met d'ailleurs en doute la pertinence d'une proposition des travailleurs visant à ajouter au préambule que les mesures en vue de la formalisation doivent être prises «tout en garantissant la préservation et l'amélioration [des] moyens de subsistance», celle-ci estimant que "quand les travailleurs auront rejoint l'économie formelle leurs moyens de subsistance s'amélioreront sans aucun doute» (CIT, 2015, paragraphe 70). La proposition des travailleurs est toutefois adoptée.

14. Des répondants représentant des employeurs de Namibie, du Sénégal ou de République de Corée soulignent d'ailleurs en vue des discussions de 2015 l'absence d'une définition de l'économie formelle et demandent des précisions sur les «dispositions formelles» visées dans la définition générale du projet, ce à quoi le Bureau répond par un renvoi aux conclusions précitées de 2002 (BIT, 2015a: 7-18). 
telles à côté de différentes dispositions. Par exemple, dans la section II du projet concernant les objectifs et principes généraux de la recommandation, le point 12 sur lequel nous nous sommes arrêtés précédemment énumère trois enjeux:

a) faciliter la transition des travailleurs et des unités économiques de l'économie informelle vers l'économie formelle (...); b) promouvoir la création d'emplois décents, leur préservation et leur pérennité dans l'économie formelle, ainsi que la cohérence entre les politiques macroéconomiques $(. .$.$) ; c) prévenir l'informalisation des$ emplois de l'économie formelle. (CIT, 2014: 149) ${ }^{15}$

Dans la section III visant le cadre juridique et politique qu'il faut dans l'ensemble réexaminer et compléter grâce à l'élaboration d'un «cadre de politiques intégrées », on remarque que la majorité des points à aborder pourrait aussi conditionner l'existence même d'une économie formelle. Il y a tout d'abord, par exemple, la création d'emplois de qualité dans l'économie formelle, le cadre législatif et réglementaire, le respect et la promotion des principes et droits fondamentaux au travail, ou la reconnaissance de l'entrepreneuriat dans l'économie formelle. Il y a ensuite les garanties d'accès à l'éducation, aux financements et à l'activité économique puis les mesures d'application avec le contrôle, l'inspection, l'accès à la justice et la coopération internationale ${ }^{16}$. Peu de points dans cette liste réfèrent à l'idée de transition précisément alors que celle-ci est étayée dans une autre disposition qui concerne la réduction des obstacles à cette idée (enregistrement, imposition et application des lois), les incitations et la promotion de la transition, les populations les plus exposées (femmes, jeunes, migrants, peuples tribaux, etc.) ou la préservation et le développement du potentiel que recèle l'économie informelle.

La section $\mathrm{V}$ sur les droits au travail ${ }^{17}$ est également illustrative car les points 20 à 22, qui promeuvent de manière générale une extension et la promotion des droits fondamentaux au travail, la sécurité et la santé au travail et leur contrôle, sont suivis du point 23 commençant par l'expression «en ce qui concerne la transition de

15. Voir le point 1 . b) et c) de la nouvelle recommandation dont le deuxième a été légèrement modifié puisqu'il s'agit de promouvoir la création d'emplois décents mais aussi d'entreprises, terme d' «entreprises» qui a donné lieu à des discussions aboutissant à un accord général sur le caractère adapté de ce mot dès lors que l'on parle de l'économie formelle (CIT, 2015: 17-18).

16. Cette disposition correspond au point 11. a) à t) de la récente recommandation. Des formulations ont été précisées étant donné qu'il ne s'agit plus d'une liste des thématiques à «aborder» mais de celle des objets sur lesquels devraient «porter» les «politiques intégrées». On passe par exemple de «l'environnement des entreprises et de l'investissement» à «la promotion d'un environnement propice aux entreprises et à l'investissement». On ne mentionne plus «l'accès au financement et au crédit» mais de manière plus générale «l'accès aux services financiers", de la même façon que l'on remplace le «contrôle de la sécurité et de la santé au travail» par «des politiques effectives» en ce domaine. Le point litigieux en 2014 relatif à l'accès réglementé aux ressources naturelles «aux fins de subsistance» est réglé par l'ajout de «publiques» après ressources naturelles.

17. Elle correspond à la partie $\mathrm{V}$ « droits et protection sociale», assez similaire, de la nouvelle recommandation. Celle-ci est précédée d'une partie IV concernant les politiques de l'emploi visant «l'objectif de créer des emplois de qualité dans l'économie formelle» et déterminant les éléments que les membres peuvent éventuellement inclure dans la détermination d'un «cadre global de politiques de l'emploi». 
l'économie informelle vers l'économie formelle ${ }^{18}$ ». Ici sont préconisées des mesures davantage progressives, concentrées sur l'extension adaptée de la protection sociale ou sur les services de garde et financiers. Le point suivant mentionne que de telles mesures progressives ne sont toutefois pas adaptées au «contexte du travail non déclaré», comme «forme d'économie informelle», qui appellerait des approches plus coercitives ${ }^{19}$.

Ainsi, à l'issue de cette rapide lecture du projet, on peut avoir l'impression que la résolution du problème de l'économie informelle doit en réalité prioritairement passer par le traitement de l'économie formelle, dont il faut revoir, voire créer, le cadre général. Mais on repère en même temps que s'il faut revoir, recréer, créer parfois ce cadre, il convient aussi d'en étendre ou adapter des aspects à l'économie informelle, alors principalement traitée dans sa dimension «secteur», appuyant du même coup certains doutes exprimés par des discutants du projet sur la teneur de ce qui constitue la finalité de la recommandation ${ }^{20}$.

Les aspects du programme sont si nombreux que l'on peut se demander si l'OIT ne pose pas un problème de l'informel dont la solution passerait par une sorte de «table rase» politique et juridique tant il est difficile de définir cette notion d'informel en elle-même. Mais il faut aussi agir vite selon le projet de préambule qui «devrait reconnaître la nécessité pour les États membres de prendre d'urgence des mesures appropriées permettant la transition des travailleurs et des unités économiques de l'économie informelle vers l'économie formelle» (CIT, 2014: 148) ${ }^{21}$. Le résultat de la concertation pose alors la question du choix de son point de départ, à savoir l'informel dans son sens le plus large et complexe, comme s'il n'y avait dans le fond plus rien, ou comme si le discours était parvenu à « [opérer], sans avertissement ni précaution, une généralisation au terme de laquelle «l'informalité» devient la propriété essentielle de la totalité [des] pratiques» (Nélisse, 1994).

Une telle lecture du projet donne alors à réfléchir de façon intéressante sur l'évolution de cette notion d' «économie informelle» qui s'est stabilisée dans les discours, s'est diffusée au Nord et a même pu être «codifiée» au Sud à force de constituer un objet de politiques publiques, aboutissant à déplacer l'enjeu de l'usage des termes formels et informels devenu "principe classificatoire simple et suffisant» vers l'État dans son articulation à la société (Nélisse, 1994). L'économie informelle devrait deve-

18. Formule remplacée au point 18 de la recommandation 204 par l'expression « dans le cadre de la transition vers l'économie informelle», reprise au point 20 sur l'extension progressive de la couverture sociale.

19. Mis entre crochets en 2014 faute de consensus sur sa place et son contenu dans la norme, ce texte ne figure plus au sein de la recommandation mais se retrouve au sein du point 22 (dans la partie VI du texte «mesures incitatives, conformité et mise en application») référant à l'application de la loi et à des sanctions effectives en cas, notamment, d'évasion fiscale ou de contournement de la législation sociale.

20. Voir notes 13 et 14 supra.

21. Des mesures immédiates sont préconisées «afin de remédier aux conditions de travail dangereuses et insalubres qui caractérisent souvent le travail dans l'économie informelle» (CIT, 2014: 152), disposition figurant désormais au point 17. a) de la recommandation 204. 
nir aujourd'hui le point de départ des politiques et réformes des régimes du travail en Afrique puisqu'elle en constitue la principale économie (main economy) (Tsikata, 2011). L'informel comme adjectif ou l'informalisation comme processus servent par ailleurs à caractériser les modalités contemporaines de l'intervention publique, dans leurs logiques partenariales au Nord (Nélisse, 1994) ou selon une forme de «gouvernement de l'informel» en Amérique latine (Lautier, 2003). En Afrique, l'informalisation travaille au cour de la vie sociale: «on retrouvera cette informalité non seulement dans les domaines de l'économique, mais au cœur même de l'État et de l'administration et dans bien des secteurs de la vie sociale et culturelle ayant quelque rapport avec la lutte pour la survie (...). Elles deviendront petit à petit les formes privilégiées de l'imagination culturelle et politique» (Mbembe, 2010: 193-194).

Si l'OIT tient alors compte dans sa démarche normative de l'infinie complexité de la question qui est posée, l'initiative politique soulève celle de ses retombées réelles, à la fois sur le plan pratique et sur celui de sa diffusion. Ne repère-t-on pas ici le choix d'une thématique générale qui permet de poser un problème, et d'agir dessus, sans avoir à en préciser vraiment les termes? On voit en effet à la lecture des différents rapports issus du processus international que si l'informalité est partout présente, certains aspects du problème ne sont pas aussi facilement abordables à l'OIT comme l'atteste par exemple le débat irrésolu en 2014 sur la mention ou non des chaînes mondiales d'approvisionnement et de la sous-traitance dans la future recommandation ${ }^{22}$.

Cet exemple, auquel on pourrait ajouter celui des discussions quant aux choix terminologiques à faire entre «unités économiques» et «entreprises», illustre en effet la relative indétermination du champ que cet instrument est appelé à couvrir. Car si le problème de l'économie informelle semble presque embrasser celui de l'«informalisation de la société» (Lautier, 2004), le découpage entre l'informalité des unités et l'informalité du travail favorise également un objet d'intervention politique tout à fait malléable, en particulier du point de vue du droit ${ }^{23}$. Or, une «économie informelle» n'est-elle pas constituée précisément là où est mis en place

22. La formule «y compris dans le cadre de la sous-traitance et des chaînes d'approvisionnement » placée entre crochets au point 9 e) du projet a soulevé un débat qui n'est pas encore réglé à l'issue des discussions en 2014 faute d'un consensus entre tous les groupes, le groupe des employeurs considérant que l'on sort avec cette mention de l'objet de la recommandation (CIT 2014: 149 et 56-60; BIT 2015a). Le point 4 c) de la recommandation précise désormais, à la suite des «consultations informelles ultérieures [ayant] permis de progresser vers un consensus sur le libellé (...)» (CIT, 2015) que la recommandation s'applique "aux salariés ayant des emplois informels au sein des entreprises formelles ou des unités économiques de l'économie informelle, ou travaillant pour elles, notamment dans le cadre de la sous-traitance et des chaînes d'approvisionnement».

23. La comparaison de l'ancienne et de la nouvelle version des paragraphes précisant le champ d'application de la norme (points 8 et 9 en 2014 et point 4 en 2015) montre d'ailleurs, malgré un effacement de la séparation explicite entre unités et emplois, un renforcement de la distinction de ces deux pans de l'économie informelle qui ne semblent plus se chevaucher. 
par le droit de la $\mathrm{ZFE}^{24}$ un espace exonéré de procédures et formalités communes au reste du pays? Ou bien, l'informalité se situe-t-elle davantage là où la présence institutionnelle s'exerce «sans formes» mais quotidiennement?

L'étude de la manifestation de la forme du droit dans ce que nous choisissons d'appeler dans le contexte du Togo deux économies informelles laisse ces questions relativement sans réponse car même si elle confirme que le droit est bel et bien ambivalent dans l'économie informelle, elle montre aussi une question de l'économie informelle aux points d'entrée extrêmement divers en ce qui concerne cet objet d'intervention politique et juridique.

\section{PRÉSENCES dU DROIT dANS DEUX ESPACES D'ÉCONOMIES INFORMELLES AU TOGO}

On peut repérer du point de vue du droit togolais deux espaces juridiques de l'économie informelle. D'un côté, il y a la ZFE et de l'autre le «secteur informel $»^{25}$ que l'on peut qualifier d'officiel au sens où il fait l'objet en tant que tel de réglementations et d'institutions particulières. Ces deux espaces renvoient ainsi à deux types d'économies informelles avec des règles officielles distinctes qui encadrent et qui sont mobilisées de manière presque opposée sur le plan de l'activité économique (II.1.) et du travail (II.2.).

\section{II.1 Formes du droit et modalités d'encadrement des activités économiques}

L'étude de l'encadrement des activités économiques consiste à partir dans un premier temps du droit et des modalités de sa mise en œuvre qui déterminent les conditions de mise en place et d'exécution de l'activité de l'entreprise au Togo. Même si les ZFE

24. Il n'est pas rare qu'informel et zones franches soient abordés à côté dans les documents institutionnels de l'OIT. Il est évoqué le «déficit considérable dont pâtissent les travailleurs des zones franches d'exportation et ceux qui font vivre l'économie informelle, sur le plan de l'organisation syndicale, tant en droit que dans la pratique» (CEACR, 2009: 33). On relève souvent l'emploi informel qui est créé dans les ZFE (Car et Chen, 2002: 6) mais la zone franche en tant que telle ne fait pas partie de l'économie informelle. L'ampleur de l'économie informelle serait même une cause des mauvaises conditions de travail dans les ZFE tout en servant de justification à ces politiques. Car, selon l'OIT et l'OMC, «les avantages dynamiques que peut procurer une ZFI dépendent aussi du niveau de développement du pays, car les pays plus pauvres peuvent avoir plus de mal à absorber les innovations technologiques dans leur économie» (BIT/OMC, 2009: 123-124).

25. Différents rapports nationaux donnent l'indication d'une présence impressionnante du secteur informel au Togo. Dans le DSRP (programme PPTE) complet de 2009, on parle d'une proportion de $80 \%$ en comptant le secteur agricole (DSRP-C, 2009). La part occupée par la ZFE en résulte modeste dans l'économie togolaise avec seulement une soixantaine d'entreprises agréées en 2010. Le chiffre d'affaires d'environ 171 milliards de FCFA en 2009 touche surtout le secteur du bois et de la construction, l'industrie plastique et l'agroalimentaire pour des exportations principalement tournées vers l'Afrique (CSI/BIT, 2012). Sur 10516 travailleurs en 2010, 2725 sont des ouvriers qualifiés permanents ( 570 femmes) et 5308 des temporaires semi-qualifiés et non qualifiés (2752 femmes). Concernant la part d'informalité en ZFE, les statistiques montrent que seulement 2761 travailleurs sont déclarés à la caisse nationale de sécurité sociale et que 24 entreprises sur 62 n'en déclarent aucun (source: SAZOF, 2011). 
ne font en principe pas partie de l'économie informelle au sens de celle des unités économiques, il apparaît pertinent ici pour la comparaison entre ces deux espaces d'informalité de partir précisément de ce qui régit les activités des différents opérateurs économiques.

\section{II.1.1 Une surformalisation de l'absence de formes en ZFE}

Le droit national qui crée un régime de ZFE paraît programmer une certaine herméticité économique, mais aussi matérielle et institutionnelle de cet espace par rapport au reste du pays, ce qui peut évoquer ici un cas de surprésence du droit dans la production d'une «économie informelle» de la ZFE. La mise en place de politiques de zone franche destinées à favoriser l'investissement dans les pays en développement s'inscrit d'ailleurs aujourd'hui dans une approche internationale, et régionale ${ }^{26}$, qui paraît diffuser un certain modèle d'efficacité de cet outil en insistant particulièrement sur la réduction des contraintes formelles grâce à une gestion privatisée, une administration de type corporatif et des contrôles de douane limités (Farole, 2011; FIAS, 2008).

Si les États abandonnent des revenus fiscaux et des pouvoirs afin de doper le commerce extérieur, c'est, certes, sous la pression des contraintes de la mondialisation, mais aussi par des réglementations détachées des autres dispositifs de droit en vigueur dans le pays. Ces dispositions nationales qui créent les statuts et déterminent le régime des activités, des biens et de leur mobilité révèlent donc assez bien le type de projet économique, institutionnel et territorial qui peut être diffusé dans la société par ces politiques gouvernementales.

Concernant le Togo, schématiquement, au premier «bloc» de textes composé de la loi de 1989 et de son décret d'application de 1990 a succédé le second bloc aujourd'hui en vigueur, composé de la loi de 2011 et de son décret d'application de $2013^{27}$. Le régime des biens et de la production en ZFE n'en a pas été fortement modifié et continue de comprendre les avantages douaniers et fiscaux consentis par les pouvoirs publics, ainsi que des dispositions administratives et matérielles d'accompagnement, ou de sécurisation de l'activité exportatrice.

Le régime comporte donc d'une part des exonérations de droits de douane d'entrée et de sortie, des exonérations et réductions fiscales sur les bénéfices, les biens, les dividendes et autres aspects financiers. L'article 23 de la loi de 2011 prévoit par exemple que «les entreprises installées en zone franche sont exonérées de tous droits, impôts et taxes qui ne sont pas expressément visés aux articles 20, 21 et 22 ci-dessus »,

26. L'AFZA est l'Africa free zone association mise en place en 2004 et installée au Nigeria (voir www. afzaonline.com).

27. Loi ${ }^{\circ} 89-14$ du 18 septembre 1989 portant statut de zone franche de transformation pour l'exportation, décret $n^{\circ}$ 90/40 du 4 avril 1990, loi n 2011-018 du 24 juin 2011 portant statut de zone franche industrielle, décret $\mathrm{n}^{\circ}$ 2013-090/PR du 27 décembre 2013. Si le code des investissements de 2012 paraît réinsérer la ZFE dans le champ du droit national, c'est bien au statut de la loi de 2011 qu'il renvoie en précisant qu'il est exclusif du reste du code. 
articles 20 à 22 qui précisent le régime de l'impôt sur les sociétés, de la taxe sur les salaires, de la taxe professionnelle, de la taxe foncière et de l'impôt sur les dividendes. Une nouveauté en 2011 est la «clause de stabilité fiscale» de l'article 38, consistant à garantir qu'aucune modification ne pourrait être apportée au régime fiscal par le code général des impôts, ce qui revient à dire implicitement que l'assemblée législative n'est pas habilitée à modifier indirectement le statut de ZFE par la loi fiscale ${ }^{28}$.

Le régime de ZFE est de plus administré par la Société d'administration de la zone franche (SAZOF) qui a le statut de société d'économie mixte financée en partie par les entreprises privées $^{29}$. La loi précise toutefois que les tâches de police et de maintien de l'ordre sont en partie assurées par les forces de l'ordre publiques et le décret prévoit certaines modalités de mise à disposition des services douaniers, notamment «pour accélérer le dédouanement». S'agissant des liens avec d'autres services et productions du pays, sont garantis la liberté de se fournir ou non localement, le choix de constituer son propre réseau d'eau, d'électricité ou de télécommunication, et le bénéfice de certains tarifs préférentiels.

Il y a enfin, du fait du régime des activités, des biens et capitaux en ZFE, la création par la loi de la fiction de la nature internationale des opérations entre les zones et le territoire d'accueil. En effet, les articles 28 et 29 de la loi de 2011 établissent que, d'une part, la vente de biens et services produits en zone franche implique le paiement des droits et taxes de douane "quelle que soit l'origine des matières premières» et que, d'autre part, «les ventes à destination des entreprises admises au statut de zone franche, réalisées par des entreprises installées sur le territoire douanier, sont considérées au titre de la réglementation comme des exportations».

Ainsi, on repère un encadrement qui organise la coupure de ces espaces des zones franches avec le reste du pays d'accueil, sur le plan douanier et plus largement institutionnel et matériel. Or, le fonctionnement autonome que suggère le régime de l'activité et que justifie la mobilité des biens et capitaux n'est-il pas un aspect important des questions d'informalité dans et autour de la ZFE?

Pour prendre un exemple concret d'informalité créée par ce régime, on peut citer le propos d'un représentant syndical des commerçants du nord du pays qui précise " [acheter] les mèches, les éponges en fer, certains pagnes, des produits cosmétiques de la zone franche» et évoque certains problèmes en ce qui a trait aux services douaniers: «nous n'avons pas d'avantages spécifiques à la douane, nous avons seulement des indulgences ${ }^{30} »$. À côté des effets de contournements de ce type favorisés par le

28. Ce type de clause inséré dans les codes des investissements africains fut critiqué au lendemain des indépendances (Kahn, 1965).

29. Décret $n^{\circ}$ 94/PR du 16 mars 1994 portant création de la SAZOF. La SAZOF devrait être dissoute dans la future Agence nationale de la promotion des investissements et de la zone franche (API-ZF) qui aura le statut d'établissement public autonome. Le décret de mise en place de cette autorité est publié au journal officiel depuis le 31 janvier 2014 mais la SAZOF se charge encore du régime aujourd'hui comme peut d'ailleurs l'illustrer la présence de trois de ses représentants à titre de conseillers techniques gouvernementaux à la CIT de juin 2015.

30. Entrevue à Dapaong, région des savanes, 24 janvier 2009. 
régime, comme c'est le cas d'ailleurs de toute loi et, qui plus est, dans le contexte du Togo, la loi paraît produire ici un espace d' «économie informelle» dont les enjeux tiennent visiblement à la légalité «formelle» elle-même de sa délimitation institutionnelle en matière économique.

\section{II.1.2 Le cadre et les modalités de l'intervention publique quotidienne dans le «secteur informel»}

Les composantes du cadre formel qui participent à produire une autonomie «informelle» et fonctionnelle de la ZFE font assez bien écho aux modalités d'interventions publiques dans les activités économiques du «secteur informel» de ce pays. Ces interventions concernent d'ailleurs principalement le droit fiscal local ou étatique et la douane.

Sur le plan fiscal, les pressions et harcèlements quotidiens relatés par certaines personnes contrastent particulièrement avec le caractère de "paradis économique ${ }^{31}$ » de la ZFE où l'on n'imagine mal des contrôleurs fiscaux procéder à ce type de visite. L'informel serait donc ici surcontrôlé, en particulier la partie de celui-ci qui s'exerce dans un établissement ou à un endroit fixe de l'espace public.

Parmi les contributions fiscales que les unités du secteur informel peuvent être amenées à payer, il y a les perceptions de taxes municipales couramment appelées le «ticket» pour tout ce qui concerne l'utilisation de l'espace public. Certains mentionnent également le passage du service de contrôle des prix qui vérifie les produits. Il y a en outre et surtout le «régime d'imposition synthétique dénommé régime de taxe professionnelle unique (TPU)» réglementé par le Code général des impôts du Togo depuis la fin des années 1990. En vertu de ce programme d'imposition simplifiée, toute personne physique est en principe assujettie à cet impôt au titre de ses activités professionnelles dès lors que le chiffre d'affaires ne dépasse pas 30 millions de FCFA pour les opérations de production et de livraison de biens ou 10 millions de FCFA pour le reste. Cette taxation concerne donc indifféremment les activités sous ce plafond, qu'elles soient rémunératrices ou non. L'impôt est fixé à partir du chiffre d'affaires évalué sur la base de la déclaration du contribuable ${ }^{32}$ en fonction d'un taux de 2,5\% dans la production et le commerce et de $8,5 \%$ pour les services. Il y a même un barème «pour les professions exercées en ambulance» établi selon le moyen de déplacement:

- Véhicule automobile (par véhicule)................ 50000 FCFA

- Véhicule à moteur à deux ou trois roues ou charrette (par engin). . 15000 FCFA

- Vélo (par vélo) ........................... 6000 FCFA

- Autres moyens ........................... 5000 FCFA

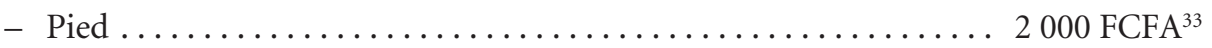

31. Expression utilisée par un syndicaliste (entrevue à la CSTT, Lomé, 25 et 28 novembre 2008).

32. Avant cet article L5 du Livre des procédures fiscales de 2014, on parlait d'un «chiffre d'affaires estimé par l'administration fiscale».

33. Article 1429 b) du Code général des impôts du Togo (voir les documents en ligne sur togo. eregulations.org). 
La mise en œuvre de ces dispositions qui visent à prendre en compte le «secteur informel» à partir d'obligations comptables « réduites à la tenue d'un livre de recettes et de dépenses» n'est pas sans poser de nombreux problèmes. Ils concernent la base de l'évaluation du montant d'impôt, certaines modalités violentes d'application et l'absence d'investissements publics visibles en retour. Un gérant d'atelier de menuiserie à Lomé raconte par exemple en 2009 que «les impôts aussi viennent si je ne suis pas en règle. Ils viennent pour fermer. Ils sont déjà venus, on a négocié, ils font l'effort quand même de négocier. Les impôts, ça fait souvent 10000 par $\mathrm{an}^{34}$.» Selon un commerçant du nord du Togo, le service des impôts « connaît presque toutes les boutiques et même les revendeuses, pour le paiement des impôts. (...) Ils ont des critères à leur niveau, ils dépendent de la taille de l'établissement. Car une revendeuse de tomates ne peut pas payer le même prix qu'un boutiquier. Il faut noter que le plus souvent, ils n'ont pas une idée arrêtée du prix et c'est souvent sur le terrain qu'ils se mettent d'accord, trouvent un consensus ${ }^{35}$.»

Si le traitement fiscal du secteur informel semble dans l'ensemble individualisé et négocié de personne à personne, certains évoquent des mouvements collectifs qui visent à contester l'impôt forfaitaire et les droits de douane. Certains représentants syndicaux mentionnent par exemple leur intervention dans le problème de la TPU afin de mieux informer les contribuables ${ }^{36}$. Un autre précise que «les femmes se rebellent contre les percepteurs des taxes, contre l'insalubrité, vous voyez chaque matin une femme paye $150 \mathrm{~F}$ au moins. Qu'est-ce qu'on fait avec l'argent ${ }^{37}$ ?» Il y a également des problèmes concernant les douanes aux frontières du Togo. Le commerçant précité du nord du pays parle de «mouvements» ou «soulèvements» rattachés notamment au régime d'exception de la zone franche car «on permet une importation hors taxe, mais ces produits n'ont plus le droit de revenir dans le pays ${ }^{38} »$.

On a donc l'impression que le traitement public du «secteur informel» est quotidien, au contraire d'un sentiment d'im-pénétration de certains espaces comme celui des ZFE. Le droit est bien présent dans les deux cas dans les réglementations officielles et les modalités de leur mise en œuvre institutionnelle. La comparaison entre ces deux économies informelles, mais aussi les liens entre elles, renseigne en outre sur un droit officiel de «l'ensemble de la société» impliqué dans la question informelle au Togo.

34. Entrevue à Lomé, 29 janvier 2009.

35. Entrevue à Dapaong, région des savanes, 24 janvier 2009.

36. «Il y a eu des émissions sur les radios privées et autres, nous avons fustigé les comportements des agents des impôts, même de la direction générale des impôts... et cette année, ils ont initié déjà des séquences de sensibilisation» (entrevues à Lomé, 25 et 28 novembre 2008).

37. Entrevue à Lomé, 23 décembre 2008.

38. Entrevue à Dapaong, région des savanes, 24 janvier 2009. 


\section{II.2 Les applications ou implications du droit du travail dans l'économie informelle}

Un contraste entre ces deux économies informelles est également repérable en matière de droit du travail entre la garantie juridique d'une certaine autonomie de fonctionnement en ZFE et des modalités aléatoires de l'intervention du droit dans le «secteur informel».

\section{II.2.1 Le remplacement de règles illégales par des procédures propres à la ZFE}

Les controverses sur les droits des travailleurs dans les ZFE sont particulièrement bien illustrées par le cas togolais. La création formelle d'un droit dérogatoire réservé aux employeurs de la ZFE qui a favorisé plus de vingt ans de silence syndical, judiciaire et administratif illustre assez bien ce pan de l'économie informelle de la non-couverture, «en droit», par des dispositions formelles. La réforme de la loi de zone franche en 2011 a permis l'affirmation officielle du retour de la ZFE dans le champ d'application du droit du travail. Toutefois, la question de l'institution d'une frontière entre la zone et le pays d'accueil vectrice de l'informalité de la ZFE peut encore être posée aujourd'hui.

Jusqu'à la fin des années 2000, le premier bloc de textes qui encadrent la ZFE, complété par un accord collectif en 1996, va programmer un droit du travail dérogatoire. La loi de 1989 affirme de façon générale «la liberté d'embauche et de licenciement du personnel togolais ou expatrié» tandis que le décret de 1990 détaille un régime spécifique du travail, en précisant: 1 . Les dispositions du Code du travail qui s'appliquent; 2. Le principe général de la libre négociation des conditions de travail; et 3. La non-application des "procédures et formalités» prévues par le droit du travail pour le recrutement, le licenciement individuel et collectif, les contentieux individuels et collectifs, et la classification professionnelle. Un accord collectif adopté en 1996 va ensuite appuyer cet aspect procédural en confirmant le règlement définitif de tout différend de travail dans la zone. On réinstaure donc des procédures en ZFE mais elles sont programmées pour se dérouler exclusivement dans son cadre puisque la SAZOF se substitue de manière générale à l'administration publique et aux instances judiciaires (Panier, 2014: 331-362).

Ces textes, par ailleurs silencieux sur le syndicat et la grève, vont favoriser une longue absence des acteurs publics et syndicaux en zone franche ${ }^{39}$. Mais une telle situation n'est plus tenable à la fin des années 2000, du fait de la pression internationale $e^{40}$, de celle d'acteurs collectifs associatifs ou syndicaux et même de la population

39. À côté des raisons politiques de cette absence, il y a l'utilisation de l'argument juridique. On peut citer un jugement du Tribunal du travail à Lomé du 20 mars 2001 (inédit $n^{\circ}$ 031/2001) qui déclare une requête irrecevable en se fondant sur le «caractère obligatoire» des procédures spécifiques contenues dans la loi de 1989, le décret de 1990 et l'accord collectif de 1996.

40. La Commission d'experts pour l'application des conventions et recommandations (CEACR) de l'OIT chargée du suivi du respect des normes internationales du travail par les États n'a par exemple pas cessé depuis 1995 de formuler des observations et demandes au gouvernement du Togo sur la syndicalisation en ZFE (base de données normlex de l'OIT). 
dans son ensemble ${ }^{41}$. La syndicalisation s'amorce en 2009 et la nouvelle loi sur la ZFE du 24 juin 2011 affirme que «les dispositions du Code du travail s'appliquent aux entreprises agréées au statut de zone franche». Depuis le mois d'octobre 2012, la ZFE constitue en outre un secteur régi par «la convention collective de la zone franche du Togo » signée par les trois syndicats désormais en place et l'association patronale de zone. Cette convention ne contient plus le remplacement des autorités judiciaires et administratives par la SAZOF et n'évacue plus le syndicat et la grève de son domaine.

On voit dès lors assez bien le processus à l'œuvre de formalisation du travail en zone franche du Togo à partir des réformes et de la reconnaissance syndicale dans cet espace. Néanmoins, ce processus comporte certains indices d'une possible continuité d'un traitement différencié du travail pouvant freiner certaines retombées pratiques sur l'emploi informel dans les entreprises de ZFE. On peut par exemple citer le débat perceptible sur le plan judiciaire, à un moment où la réforme à venir de la loi ne fait plus trop de doute, et qui concerne son effet d'annulation ou non de l'accord de $1996^{42}$, débat que la décision de la SAZOF de n'étendre en 2012 qu'une partie de la Convention collective interprofessionnelle du Togo signée en 2011 viendra en quelque sorte trancher ${ }^{43}$. La convention collective de la zone franche en vigueur depuis 2013 réfere en outre à un inspecteur du travail dit «du ressort» de la ZFE en tant qu'autorité compétente sur de nombreux points; autorité qui semble correspondre à l'inspecteur du travail du secteur public qui, au moment des réformes, n’a pas été juste désigné mais transféré, physiquement et juridiquement, au sein de la SAZOF. Il y a aussi les mentions «vu» par le directeur général du travail et celui de la SAZOF et «approuvé» par le ministre chargé du travail et par celui chargé de la zone franche au bas de la nouvelle convention.

Ainsi, il peut être intéressant de se pencher, par-delà la distinction entre l'emploi informel des travailleurs et le secteur informel des unités telle qu'établie dans le cadre de l'OIT, sur les manières par lesquelles le droit, à partir du syndicat, du juge ou de l'inspecteur du travail, entre dans l'espace institutionnel spécifique de la ZFE dans le cadre de cette transition vers la formalité amorcée à la fin des années 2000.

Concernant par exemple la syndicalisation des travailleurs, les trois syndicats présents étant affiliés à des centrales syndicales nationales, on peut considérer qu'on a assisté au retour de la zone dans le champ du droit syndical togolais. Certains indices permettent toutefois de relever une entrée syndicale possiblement inscrite dans la spécificité institutionnelle de la zone. Des décisions sont par exemple encore rendues dans le cadre de la procédure de règlement des litiges à la SAZOF alors que des syndicats sont désormais en place ${ }^{44}$. On peut aussi citer l'utilisation syndicale du siège de

41. Les journées portes ouvertes de la ZFE organisées à la fin de 2008 ont attiré beaucoup de visiteurs dont les questions abordaient surtout les conditions de travail.

42. Entrevues au Tribunal du travail de Lomé, décembre 2008 et janvier 2009.

43. Voir par exemple, le lien www.ituc-csi.org/togo-1e-mai-2012-application-du? lang=fr ou www. republicoftogo.com/Toutes-les-rubriques/Social/Zone-franche-discussions-sans-pressions

44. Conseil d'interprétation et de conciliation (composé de représentants de la SAZOF, des employeurs et de délégués du personnel), PV des $1^{\text {er }}$ décembre 2010, 18 et 25 janvier 2011. 
l'autorité de zone pour l'organisation d'une «session de renforcement des capacités au profit des employeurs et des travailleurs des unités de production de la zone franche $»^{45}$. L'actualité récente concernant une grève commune de travailleurs membres d'un syndicat sectoriel des mines pour dénoncer les pratiques de trois sociétés, dont deux appartenant à la zone franche, peut être à l'inverse analysée comme un indice d'inscription du problème de zone franche en dehors de son espace "réservét6"

Ce récent conflit concerne notamment le recours d'une société de la ZFE à un sous-traitant (tâcheron) pour l'utilisation de centaines de travailleurs ${ }^{47}$, une pratique qui s'ajoute à la gestion par statuts de travail que l'on pouvait déjà noter en zone franche avant les réformes et la syndicalisation (voir Lendro, 2014: 576-582). Il faudrait établir si certaines violations des droits des travailleurs ne sont pas justement favorisées par les modalités d'institutionnalisation du périmètre de la zone franche dès lors qu'elles reconduisent une gestion relativement indépendante des questions de travail dans la zone. On retrouve alors ici la question de la part d'autonomie des autorités de ZFE, en droit et en pratique, pour aborder l'informalité dans cet espace où les unités sont formelles et l'emploi est informel.

\section{II.2.2 Présences juridiques et institutionnelles du droit du travail dans l'économie informelle «officielle»}

Dans l'économie informelle «officielle» du Togo, on trouve quelques règles formelles spécifiques mais elles sont assez rares. Il y a par exemple des dispositions particulières dans le code de la sécurité sociale de 2011 qui prévoient l'application de certaines protections sociales ${ }^{48}$, conformément aux préconisations internationales en matière de formalisation (CIT, 2014: 156) ${ }^{49}$. La présence du droit social dans cette économie informelle tient donc davantage aux mobilisations de celui-ci sur le plan étatique et syndical. À partir de l'enquête auprès de différents acteurs togolais, on peut en effet relever que c'est moins le manque de dispositions juridiques formelles adaptées à l'économie informelle qui pose problème sur cette question que l'informalité en ellemême dans sa réalité quotidienne, socioéconomique et institutionnelle.

En ce qui concerne l'extension des protections du Code du travail aux travailleurs de l'économie informelle, des pratiques et interventions institutionnelles justifiées par la définition juridique du travailleur constituent autant de manifestations du droit du

45. Actualité en ligne sur le site cssttogo.org, novembre 2013.

46. http://news.alome.com/h/36017.html; www.togosite.com/index.php/societe/620-togo-les-travailleurs-de-wacem-fortia-et-paper-bag-crient-leur-ras-le-bol

47. www.peuples-solidaires.org/togo-zone-de-non-droit-pour-les-travailleurs/

48. Loi n ${ }^{\circ}$ 2011-006 portant code de sécurité sociale au Togo, JO 21 février 2011, p. 11. L'article 3 par. 3 prévoit que sont assujettis «pour les branches des pensions et des prestations familiales, les travailleurs de l'économie informelle». Peu de précisions sont apportées dans le reste du document sur l'application.

49. Voir les points 18 à 20 de la recommandation 204 concernant l'extension progressive de la protection sociale et, en particulier, le troisième envisageant l'adaptation des procédures, prestations et contributions. 
travail dans l'informel ${ }^{50}$, aussi bien pour disqualifier que pour qualifier la relation de travail. Paradoxalement, mais aussi à l'instar de certaines analyses juridiques de la question $^{51}$, c'est la Délégation à l'organisation du secteur informel (DOSI) créée en 2008 et «visant à recenser toutes les activités relevant du secteur informel, organiser le secteur, proposer les textes législatifs et réglementaires devant régir le secteur et, contrôler et réguler ses activités ${ }^{52}$ » que l'on affirme que «si on est dans le cadre du secteur informel, il n'est pas salarié ${ }^{53}$ » car parler de «salarié» signifierait que la régulation se fait d'ores et déjà par le droit, que le droit encadre ce secteur.

Cette approche de l'autorité dédiée au «secteur informel» contraste avec les considérations d'autres fonctionnaires selon lesquelles le droit doit être accommodé dans les contextes de l'informel. Un magistrat de Lomé dit par exemple de manière inversée qu’il «applique [les faits] au droit positif ${ }^{54}$ ». Il insiste sur le critère du «lien de subordination entre un employé et un employeur» et relève «beaucoup d'affaires surtout dans le cas des domestiques» ou des «cuisinières dans les restaurants et les jeunes qui vendent au grand marché chez les grandes dames». Un inspecteur du travail de région considère plutôt pour sa part que «dans le secteur informel, on regarde les trois conditions mais en général il n'y a pas cette subordination». Il ajoute toutefois que "quand les problèmes surgissent, ils viennent ici et on constate qu'il y a contrat ${ }^{55}$ ».

Des notions juridiques inspirées du système formel de droit du travail togolais comme celles de la "pré-conciliation», du «contrat tacite», de la "prime de bonne séparation» ou de la "réintégration» sont utilisées pour préciser les modalités de leur intervention. Certains affirment par ailleurs déborder le champ de leur compétence en expliquant par exemple «tout juste aider» les conducteurs de taxis-motos en cas de litige. Des magistrats du nord du pays, incompétents en principe en matière sociale, disent pour leur part intervenir, plus ou moins formellement, pour des litiges du travail. Beaucoup de fonctionnaires insistent en dernière analyse sur la question du revenu et se disent méfiants concernant une réglementation qui viendrait encadrer le «secteur informel» en matière de droit du travail:

Si vous demandez au propriétaire de payer $28000 \mathrm{~F}$ à la personne qui se charge de cette cabine, elle va fermer. Il y a aussi les bars. Je crois qu'à ce niveau on essaie de voir l'employeur, de voir si raisonnablement il peut payer ce SMIG. Vous voyez puisque celles qui pilent, elles sont nombreuses, trois par exemple... C'est très complexe. On réfléchit mais... C'est pour ça que je ne m'avance pas trop à toucher le secteur informel ${ }^{56}$.

50. Certains y voient des innovations juridiques récentes à partir du projet OHADA (Lager, 2009: 85) mais il faut préciser que la définition est la même au Togo depuis le Code de 1952 (Panier, 2014: 74).

51. Voir note 3 supra.

52. Projet de politique nationale du travail, Lomé, 2010.

53. Entrevue à la DOSI, Lomé, 30 décembre 2008. Pourtant selon le site de la DOSI, le secteur informel désigne «l'ensemble des activités économiques qui sont inconnues de la comptabilité nationale (mais pas nécessairement du fisc) », à savoir «l'ensemble des activités de production, de service et de commerce traditionnel, réalisées du reste avec un salariat limité et un capital relativement faible» (www.dositogo.org).

54. Entrevue au Tribunal du travail, Lomé, 3 février 2009.

55. Entrevue à Atakpamé, Plateaux, 15 janvier 2009.

56. Entrevue collective avec de jeunes inspecteurs du travail, Lomé, 16 décembre 2008. 
Cette idée d'intervention souple et compréhensive des fonctionnaires apparaît toutefois moins présente dès lors justement qu'il est question d'argent, en particulier en ce qui concerne la sécurité sociale. Selon un inspecteur du travail par exemple, «la Caisse de sécurité sociale ne fait plus la distinction entre le formel et l'informel. Quand elle descend dans la boutique, elle demande s'il y a des travailleurs, elle fait la déclaration et le reste, ça continue ${ }^{57}$.» Cette «descente» rappelle ce qui se serait produit au moment d'une opération nationale en 2008 qui aurait conduit à de nombreuses cessations d'activités (Panier, 2014: 498-500): «Quand l'État intervient, on pense que c'est pour des impôts ${ }^{58}$.»

L'argent est également une donnée problématique concernant une autre manifestation du droit du travail, à savoir l'organisation de certains secteurs en syndicats de base rattachés à des centrales nationales ${ }^{59}$. On peut repérer deux dimensions en pratique de la représentation et du soutien des membres de ces organisations qui rendent assez ambigu le rôle des syndicats en matière d'économie informelle. D’un côté, le syndicat offre certains services sociaux et propose des plateformes de concertation sur certaines problématiques politiques, économiques et sociales. D’un autre côté, il arrive que le syndicat se comporte en gestionnaire de type «patronal», voire participe à la perduration de certains rapports d'exploitation entre les employeurs de l'informel et leurs apprentis. Ainsi, entre précarité sociale à soutenir dans ce contexte socioéconomique général et manne financière non négligeable, la représentation syndicale de l'informel est remplie de paradoxes.

Le syndicalisme togolais rassemble de façon intéressante dans des fédérations sectorielles des membres de syndicats de base qui peuvent être aussi bien des travailleurs déclarés d'une entreprise inscrite au registre du commerce que des travailleursemployeurs indépendants ou membres de coopératives de l'informel. Ce type de fédération conduit à des rassemblements syndicaux où des thématiques générales comme la "vie chère», les droits humains ou la société civile sont au centre des discussions et permettent de mettre ensemble différents pans d'un même contexte socioéconomique et sociopolitique.

Par ailleurs, des syndicats offrent certains services sociaux comme des accès à des soins de santé via la mise en place de centres et de mutuelles de santé ou des formations aussi bien syndicales que professionnelles. Même des centrales syndicales qui n'embrassent pas en principe les unités économiques de l'informel participent à la mise en place d'activités, comme des formations en agriculture en ce qui concerne un syndicat de cadres, ou la création d’une «activité parallèle génératrice de revenus » par

57. Ibid.

58. Entrevue avec un inspecteur du travail, région de Kara, 6 décembre 2008. D’après le rapport de l'opération, ce serait seulement 81 employeurs qui auraient été prospectés par hasard, pour un nombre de 363 travailleurs (source: CNSS, 2009).

59. Ce syndicalisme aurait été consacré avec l'article 6 du Code du travail de 2006: «peuvent également librement constituer des syndicats, les exploitants indépendants, même s'ils n'emploient pas de personnel». 
une unité syndicale locale pour les femmes salariées des écoles primaires privées ${ }^{60}$. Dans ce dernier cas, le syndicat fait plus que de la formation car il participe à la création d'une activité lucrative possiblement informelle.

Il y a ensuite des formes de syndicats «patrons» de leurs membres comme dans ce cas concernant une activité de taxis urbains gérée par une autre centrale syndicale à Lomé:

Ici nous intervenons auprès des institutions de la place pour acheter un véhicule à tel, pour le garantir, pour qu'il puisse travailler, payer. (...) Vous faites un contrat que vous signez. Et dans ce contrat-là, il a des traites à payer. Et lui, il travaille, vous le surveillez, il travaille, il paie. À la fin le véhicule devient sa propriétét

Pour finir, on note au Togo une prise en charge syndicale importante de la relation d'apprentissage entre les membres et les jeunes travailleurs. Dans ce domaine, les apprentis se retrouvent dans l'ensemble exclus de la représentation syndicale et doivent plutôt recourir à la voie associative ou judiciaire pour dénoncer des pratiques abusives que le syndicat peut laisser perdurer ${ }^{62}$. Car l'apprentissage constitue d'un côté une voie importante d'accès à un métier pour les jeunes déscolarisés et de l'autre un "commerce lucratif» pour les formateurs puisqu'au Togo, «ce n'est pas dans les clients qu'on gagne de l'argent $t^{63} »$. Les textes officiels sur l'apprentissage tiennent compte du caractère payant de la formation et établissent, par métiers, les durées de formation et différents frais, comme les frais de contrat, de formation ou d'examen ${ }^{64}$. La présence syndicale dans la régulation de l'apprentissage est limitée en principe mais continue d'être importante dans les faits. Il arrive en effet que la signature du contrat ait lieu au siège de la centrale à Lomé contre paiement ${ }^{65}$ ou que le syndicat gère l'organisation des examens. Il peut aussi exiger la tenue des cérémonies de libération ${ }^{66}$ qui conditionnent la remise des diplômes et que certains qualifient de véritable calvaire ${ }^{67}$.

Ainsi, on peut noter à travers ces divers champs d'action syndicale dans le secteur dit informel non seulement une présence forte du syndicat mais aussi l'ambiguïté de sa fonction où interferent des aspects politiques, sociaux et économiques. Le syndicat dévoile ici toute sa puissance et sa faiblesse à travers les modalités de prise en charge

6o. Entrevue à Sokodé, Savanes, 21 janvier 2009.

61. Entrevue à Lomé, 29 décembre 2008.

62. Tribunal du travail, jugement inédit du 9 mars 2010 relevant des «traitements inhumains et dégradants».

63. Entrevue avec une coiffeuse, Lomé, 10 décembre 2008.

64. Sur la «surréglementation» de l'apprentissage (Panier, 2014: 184-190).

65. V. Collectif des associations de défense et de promotion des droits de l'homme, Document de plaidoyer pour un apprentissage à visage humain au Togo, juin 2008.

66. Une loi de 1983 limitait la pratique aux «régions rurales de droit coutumier où cette pratique est d'usage courant» et un arrêté de 1989 encadrait les frais de "cérémonie de début» et de "cérémonie de sortie». Celui de 2005 ne contient plus de précision sur ces frais.

67. Entrevue avec le président d'une association de défense des apprenti (e) s, Lomé, 29 décembre 2008. Une coiffeuse raconte avoir dû cesser, sous la pression des membres de son syndicat, de remettre directement le diplôme aux apprenties sans passer par la cérémonie (Entrevue à Lomé, 10 décembre 2008). 
du secteur dit informel, à l'instar de ce qu'on a pu noter concernant l'intervention publique dans un contexte socioéconomique où l'argent détermine souvent la manière dont le droit s'étend ou non dans ce que l'on dénomme le secteur informel.

\section{CONCLUSION}

La comparaison que nous avons proposée entre deux économies informelles à partir d'une analyse du droit illustre bien un encadrement juridique ambivalent et directement engagé dans l'évolution de l'informalité du travail au Togo, qu'il s'agisse des dispositions en matière d'activités économiques ou de celles relatives au travail et à la protection sociale. Les modalités d'institutionnalisation de ces deux espaces d'informalité contrastent toutefois fortement entre, d'un côté, une abondance de dispositions formelles réservées, une sorte de dispositif formel de l'informalité du travail et, de l'autre, quelques dispositions affichant une certaine volonté politique de prise en charge mais d'application difficile, voire injuste. On repère par ailleurs des mécanismes de formalisation du travail au sens de l'extension des protections sociales plus officiels et textuels que concrets d'un côté et négociés de l'autre selon les circonstances et les problèmes de chacun.

On repère donc ici toute la complexité des aspects que vise à embrasser le projet de recommandation de l'OIT. Toutefois, l'hétérogénéité qui se vérifie dans le contexte du Togo, et l'impossibilité qui en résulte de se représenter l' «économie informelle» comme une seule réalité problématique pousse à interroger la démarche normative internationale. Car, par-delà l'affichage d'objectifs sociaux partagés par les membres tripartites à l'OIT, c'est sur une scène contemporaine du «global-politique» (Abélès, 2008) que s'est joué le choix de cette catégorie comme point d'entrée de la future norme internationale. Or, les types de politiques publiques fondées sur la lutte contre l'informalité qui pourrait être engagée sur le fondement du projet international apparaissent tout à fait divers. Si la notion d'économie informelle est de toute façon une notion politique, son usage ne conduit-il pas aussi et du même coup, à en minimiser les contours de sa question «dans l'ensemble de la société»?

Pourtant, on a vu que l'économie informelle au Togo représente beaucoup de choses sur le plan politique. Elle est à la fois une catégorie juridique en matière administrative, fiscale et de sécurité sociale, un objet de discours politique, économique et social, le lieu des interventions publiques informelles et quotidiennes sur le fondement du droit. Elle incarne en outre différentes modalités d'informalisation des autorités étatiques, que l'on soit d'un côté ou de l'autre de la frontière instituée par la zone franche. Elle donne à voir enfin la production de rapports au droit et à l'État dans la société et dans la mondialisation au Togo caractérisés par une certaine «réversibilité fondamentale des processus» (Mbembe, 2010: 191).

Ainsi, si l'économie informelle comme objet d'intervention politique apparaît pertinente puisque l'on souhaite lutter contre, selon une démarche fondée sur les droits, elle soulève aussi, du point de vue analytique, la question du rôle de l'usage de cette catégorie sur le plan stratégique et de ses enjeux sociaux et politiques. En consi- 
dérant le «global-politique» comme un «inducteur de normes, de concepts transversaux, de paramètres de discussion, de termes de négociation qui se diffusent dans les pores des sociétés et les esprits qui les gouvernent» (Abélès, 2008: 164), les premières lignes du rapport de 2014 en vue des discussions contiennent cette phrase assez significative concernant l'indétermination de l'objet de la future norme: «l'économie informelle fait référence à des situations différentes, ayant des causes différentes, posant des problèmes différents requérant des solutions différentes» (BIT, 2014a: 4).

\section{RÉSUMÉ}

En partant du récent projet de norme de l'OIT visant à favoriser la formalisation de l'économie informelle, cet article propose d'interroger la démarche institutionnelle à travers une réflexion sur le droit comme critère d'intelligibilité de la catégorie de l'informel. Sur la base d'une enquête réalisée au Togo, cette contribution présente les modalités nombreuses et complexes des manifestations du droit du travail et du droit de l'activité économique dans les deux espaces d'informalité du travail que sont la zone franche d'exportation et le secteur dénommé informel au Togo. Entre surformalisation spécifique et im-pénétration publique ou syndicale dans un cas, et intervention publique et syndicale relativement quotidienne dans l'autre, la manière de vouloir faire de l'économie informelle un objet d'intervention global, en distinguant l'emploi informel des unités économiques, n'est pas sans soulever des questions sur la pertinence de la démarche et le rôle de ce concept souvent critiqué mais, paradoxalement, d'usage permanent.

Mots clés: Togo, droit, travail, informel, zone franche d'exportation

\section{ABSTRACT}

Focusing on the ILO's recent standards project designed to encourage the formalization of the informal economy, this article sets out to question the institutional approach by reflecting on law as an intelligibility criteria of the informal category. Based on a survey carried out in Togo, this contribution presents the numerous and complex ways in which labour law and commercial law are expressed in Togo's two areas of informal work: the free export zone and the so-called informal sector. Given the specific over-formalization of the free export zone and the lack of public or union penetration in it, and the almost daily intervention of the State and unions in the informal sector, questions are raised on the relevance of designing global interventions in the informal economy, by making a distinction between informal employment and economic units, and the role of this often criticized concept that is nevertheless and paradoxically constantly used.

Key words: Togo, rights, labour, informal, free export zone

\section{RESUMEN}

Partiendo de un reciente proyecto de norma de la OIT que busca favorecer la formalización de la economía informal, este artículo propone cuestionar la iniciativa institucional a través de una reflexión acerca del derecho como criterio de inteligibilidad de la categoría de lo informal. Sobre la base de una investigación realizada en Togo, esta contribución presenta numerosas y complejas modalidades de las manifestaciones del derecho al trabajo y del derecho a la actividad 
económica en los dos espacios de la informalidad del trabajo: la zona franca de exportación y el sector denominado informal en Togo. Entre sobre-formalización específica e im-penetración pública o sindical en un caso, e intervención pública y sindical relativamente cotidiana en el otro, la manera de querer hacer de la economía informal un objeto de intervención global, distinguiendo el empleo informal de las unidades económicas, se hace necesario subrayar los cuestionamientos acerca de la pertinencia del enfoque y del papel de este concepto, con frecuencia criticado pero paradójicamente de uso permanente.

Palabras clave: Togo, derecho, trabajo, informal, zona franca exportación

\section{BIBLIOGRAPHIE}

AbÉLÈs, M. (2008), Anthropologie de la globalisation, Paris, Payot.

Bangasser, P. E. (2000), The ILO and the informal sector: An institutional history, employment paper 2000/9, Genève, BIT.

Bit (2015a), La transition de l'économie informelle vers l'économie formelle, Rapport V (2A), CIT, $104^{\mathrm{e}}$ session, cinquième question à l'ordre du jour, ILC.104/V/2A, Genève, BIT.

Biт (2015b), La transition de l'économie informelle vers l'économie formelle, Rapport V (2B), CIT, $104^{e}$ session, cinquième question à l'ordre du jour, ILC.104/V/2A, Genève, BIT.

Bit (2014a), La transition de l'économie informelle vers l'économie formelle, Rapport V (1), CIT, 103 session, cinquième question à l'ordre du jour, ILC.103/V/1, Genève, BIT.

Biт (2014b), La transition de l'économie informelle vers l'économie formelle, Rapport V (2), CIT, 103 session, cinquième question à l'ordre du jour, ILC.103/V/2, Genève, BIT.

Bтт (2013), Rapport de la Réunion tripartite d'experts: Faciliter la transition de l'économie informelle vers l'économie formelle (Genève, 16-20 septembre 2013), GB.319/INS/14/6, CA, Genève, BIT.

Biт (2002), Travail décent et économie informelle, sixième question à l'ordre du jour, Conférence internationale du travail, 90 session, Rapport VI, BIT, Genève, 2002.

Biт (1991), Le dilemme du secteur non structuré, rapport du Directeur général (partie I) à la Conférence internationale du travail, $78^{\mathrm{e}}$ session, Genève, BIT.

Віт/Омс (2009), Mondialisation et emploi informel dans les pays en développement, Genève, OIT/OMC.

CARR, M. et M. A. Chen (2002), Mondialisation et économie informelle: l'impact de la mondialisation des échanges et des investissements sur les travailleurs pauvres, Document de travail, 2002/F1, Genève, BIT.

Cissé, A. (2014), «Pour une approche plurale du droit africain», in De l'esprit du droit africain. Mélanges en l'honneur de Paul Gérard Pougoué, Kluwer Law International et CREDIJ, p. 1-23.

Сіт (2015), Cinquième question à l'ordre du jour: Faciliter la transition de l'économie informelle vers l'économie formelle. Rapports de la Commission sur la transition de l'économie informelle: compte rendu des travaux, $104^{\mathrm{e}}$ session, Conférence internationale du travail, Compte rendu provisoire $\mathrm{n}^{\circ}$ 10-2, ILC104-PR10-2-Fr, Genève, OIT.

Сіт (2014), Cinquième question à l'ordre du jour: Faciliter la transition de l'économie informelle vers l'économie formelle. Rapport de la Commission sur la transition de l'économie informelle, $103^{\mathrm{e}}$ session, Conférence internationale du travail, Compte-rendu provisoire nº11 (E), ILC103-PR11 (Rev), Genève, OIT.

Сіт (2002), Résolution concernant le travail décent et l'économie informelle, adoptée par la Conférence internationale du travail à sa 90 session, CIT90-CRP25-573-Fr, Genève, OIT.

Csi/Bit (2012), Togo: Zones franches, droits des travailleurs et stratégies syndicales, Genève, OIT (BIT/ ACTRAV).

FArole, T. (2011), Special economic zones in Africa: Comparing performance and learning from global experience, Washington, World Bank. 
Fias (2008), Special economic zones. Performance, lessons learned, and implications for zone development, Washington, World Bank Group.

Fontaine, L. et F. Weber (dir.) (2011), Les paradoxes de l'économie informelle. À qui profitent les règles?, Paris, Karthala.

Hart, K. (1973), «Informal Income Opportunities and Urban Employment in Ghana», The Journal of Modern African Studies, volume 11, n 1, p. 61-89.

Kahn, P. (1965), «Problème juridique de l'investissement dans les pays de l'ancienne Afrique française», Journal du droit international. Clunet (JDI), p. 338-390.

LAGER, F. (2009), «Le projet d'acte uniforme OHADA droit du travail du 24 novembre 2006 permettra-t-il de faire rentrer l'économie informelle dans le secteur formel? ", Penant: revue de droit des pays d'Afrique, $\mathrm{n}^{\circ} 866$, p. 85.

Lautier, B. (2013 [1992]), «Sociologie de l'emploi, informalité des relations de travail et stratégies familiales en Amérique latine», Cahiers du Genre, 2013/2, n 55, p. 199-217.

Lautier, B. (2004 [1994]), L'économie informelle dans le Tiers-Monde, Paris, La découverte.

LAutier, B. (2003), «Les limites de l'économie informelle comme alternative à la mondialisation libérale», Revue du Mauss, 2003/1 n 21, p. 198-214

Lautier, B., De Miras, C. et Morice, A. (1991), L'État et l'informel, Paris, L'Harmattan.

LAutier, B. (2013 [1984]), «Secteur informel et emploi: l'enseignement des pays sous-développés», TiersMonde, vol. 2, n 214, p. 151-157.

Мвемве, A. (2010), Sortir de la grande nuit. Essai sur l'Afrique décolonisée, Paris, La Découverte.

Miendjiem, I. L. (2014), «Réflexions sur le champ d'application du droit du travail dans l'espace OHADA», in De l'esprit du droit africain. Mélanges en l'honneur de Paul Gérard Pougoué, Pays-Bas/Bénin, Wolters Kluwer/CREDIJ, p. 451-484

NéLisse, C. et al. (1994), «Les formes de l'informel», Lien social et Politiques, n 32, p. 5-13.

Orт (2010), Étendre le champ d'application de la législation du travail à l'économie informelle. Recueil des commentaires des organes de contrôle de l'OIT ayant trait à l'économie informelle, Genève, OIT.

Oіт (1972), Employment, incomes and equality, a strategy for increasing productive employment in Kenya, Genève, OIT.

Panier, É. (2014), L'État et les relations de travail au Togo, Paris, L'Harmattan.

Tsikata, D. (2011), «Toward a decent work regime for informal employment in Ghana: some preliminary considerations ", Comparative Labor Law and Policy Journal, vol. 32, n 3, p. 311-342. 